\title{
APPLICATIONS OF BESSEL AND STRUVE FUNCTIONS ON GENERAL INTEGRAL OPERATORS
}

\author{
D. Breaz, C.-I. TĂNASE
}

Abstract. Using Bessel and Struve functions will find some univalent conditions for general integral operators. Also we obtain some particular cases.

2010 Mathematics Subject Classification: 30C45.

Keywords: Analytic functions, Struve functions, Bessel functions, Integral operators.

\section{INTRODUCTION AND PRELIMINARIES}

Let

$$
U\left(z_{0}, r\right)=\left\{z \in \mathbb{C}:\left|z-z_{0}\right|<r\right\}
$$

be the disc with center $z_{0}$ and and of radius $r$, the particular case $U(0,1)$ will be denote by $U$. Let $H(U)$ be the set of functions which are regular in the unit disc $\mathrm{U}$. Consider $A=\left\{f \in H(U): f(z)=z+a_{2} z^{2}+a_{3} z^{3}+\ldots, z \in U\right\}$ be the class of analytic functions in $U$ and $S=\{f \in A: f$ is univalent in $U\}$

Theorem 1.1. [5] Let $\alpha$ be a complex number, $\operatorname{Re} \alpha>0$, and $f(z)=z+a_{2} z^{2}+\ldots$ be a regular function in $\mathrm{U}$. If

$$
\frac{1-|z|^{\operatorname{Re} \alpha}}{\operatorname{Re} \alpha}\left|\frac{z f^{\prime \prime}(z)}{f^{\prime}(z)}\right| \leq 1
$$

for all $z \in U$, then for any complex number $\beta, \operatorname{Re} \beta \geq \operatorname{Re} \alpha$, the function

$$
F_{\beta}(z)=\left[\int_{0}^{z} t^{\beta-1} f^{\prime}(t) d t\right]^{\frac{1}{\beta}}
$$

is in the class $\mathrm{S}$. 
D. Breaz, C.-I. Tănase - Applications of Bessel and Struve functions ...

Theorem 1.2. [3] If the function $g$ is regular in $\mathrm{U}$ and $|g(z)|<1$ in $\mathrm{U}$, then for all $\xi \in U$ and $z \in U$ the following inequalities hold

$$
\left|\frac{g(\xi)-g(z)}{1-\overline{g(z)} \cdot g(\xi)}\right| \leq\left|\frac{\xi-z}{1-\bar{z} \cdot \xi}\right|
$$

and

$$
\left|g^{\prime}(z)\right| \leq \frac{1-|g(z)|^{2}}{1-z^{2}}
$$

the equalities hold in case $g(z)=\varepsilon \frac{z+u}{1+\bar{u} z}$ where $|\varepsilon|=1$ and $|u|<1$.

Remark 1.1. [2] For $z=0$ from inequality (3) we obtain for every $\xi \in U$

$$
\left|\frac{g(\xi)-g(0)}{1-\overline{g(0)} g(\xi)}\right| \leq|\xi|
$$

and hence

$$
|g(\xi)| \leq \frac{|\xi|+|g(0)|}{1+|g(0)||\xi|}
$$

Considering $g(0)=a$ and $\xi=z$, then

$$
|g(z)| \leq \frac{|z|+|a|}{1+|a||z|}
$$

for all $z \in U$.

Let us consider the second-order inhomogeneous differential equation(([7]), p.341)

$$
z^{2} w^{\prime \prime}(z)+z w^{\prime}(z)+\left(z^{2}-v^{2}\right) w(z)=\frac{4\left(\frac{z}{2}\right)^{v+1}}{\sqrt{\pi} \Gamma\left(v+\frac{1}{2}\right)}
$$

whose homogeneous part is Bessel's equation, where $v$ is an unrestricted real(or complex) number. The function $H_{v}$, which is called the Struve function of order $v$, is defined as a particular solution of (8). This function has the form

$$
H_{v}(z)=\sum_{n=0}^{\infty} \frac{(-1)^{n}}{\Gamma\left(n+\frac{3}{2}\right) \cdot \Gamma\left(v+n+\frac{3}{2}\right)} \cdot\left(\frac{z}{2}\right)^{2 n+v+1} \text { for all } z \in \mathbb{C}
$$

We consider the transformation 


$$
g_{v}(z)=2^{v} \sqrt{\pi} \Gamma\left(v+\frac{3}{2}\right) \cdot z^{\frac{-v-1}{2}} H_{v}(\sqrt{z})
$$

After some calculus we obtain

$$
g_{v}(z)=\sum_{n=0}^{\infty} \frac{(-1)^{n} \Gamma\left(\frac{3}{2}\right) \Gamma\left(v+\frac{3}{2}\right)}{4^{n} \cdot \Gamma\left(n+\frac{3}{2}\right) \Gamma\left(v+n+\frac{3}{2}\right)} \cdot z^{n}
$$

Let $u_{v}(z)=z \cdot g_{v}(z)=\sum_{n=0}^{\infty} \frac{(-1)^{n} \Gamma\left(\frac{3}{2}\right) \Gamma\left(v+\frac{3}{2}\right)}{4^{n} \cdot \Gamma\left(n+\frac{3}{2}\right) \Gamma\left(v+n+\frac{3}{2}\right)} \cdot z^{n+1}$.

Using Theorem 2.1 ([4]) for our case with $b=c=1, \kappa=v+\frac{3}{2}$ we obtain that:

Theorem 1.3. [4] If $v>\frac{\sqrt{3}-7}{8}$ then the function $g_{v}$ is univalent in $\mathrm{U}$.

The Bessel function of the first kind is defined by

$$
J_{v}(z)=\sum_{n=0}^{\infty} \frac{(-1)^{n}}{n ! \Gamma(n+v+1)}\left(\frac{z}{2}\right)^{2 n+v} .
$$

We consider the transformation

$$
f_{v}(z)=2^{v} \Gamma(1+v) z^{-\frac{v}{2}} J_{v}(\sqrt{z})
$$

After some calculus we obtain

$$
f_{v}(z)=\sum_{n=0}^{\infty} \frac{(-1)^{n} \Gamma(1+v)}{n ! \Gamma(n+v+1) \cdot 4^{n}} \cdot z^{n} .
$$

Let $h_{v}(z)=z \cdot f_{v}(z)=\sum_{n=0}^{\infty} \frac{(-1)^{n} \Gamma(1+v)}{n ! \Gamma(n+v+1) \cdot 4^{n}} \cdot z^{n+1}$

Theorem 1.4. [6] If $v>-2$ then $\operatorname{Re} f_{v}^{\prime}(z)<0$ for $z \in U_{1}(0,4(v+2))$ and $f_{v}$ is univalent in $U_{1}(0,4(v+2))$. 
D. Breaz, C.-I. Tănase - Applications of Bessel and Struve functions ...

\section{MAIN RESUltS}

Theorem 2.1. Let $\alpha, \gamma_{i} \in \mathbb{C}, \operatorname{Re} \alpha=b>0, u_{v_{i}} u_{v_{i}}(z)=z+a_{2}^{1} z^{2}+\ldots n \in \mathbb{N}^{*}, i \in$ $\{1,2, \ldots, n\}$. If

$$
\left|\frac{u_{v_{i}}^{\prime \prime}(z)}{u_{v_{i}}^{\prime}(z)}\right|<1,(\forall) z \in U,(\forall) i \in\{1,2, \ldots\}
$$

and

$$
\begin{gathered}
\frac{\left|\gamma_{1}\right|+\left|\gamma_{2}\right|+\ldots+\left|\gamma_{n}\right|}{\left|\gamma_{1} \cdot \gamma_{2} \ldots \cdot \gamma_{n}\right|} \leq 1, \\
\left|\gamma_{1} \cdot \gamma_{2} \cdot \ldots \cdot \gamma_{n}\right| \leq \frac{1}{\max _{|z| \leq 1}\left[\frac{1-|z|^{2 b}}{b} \cdot|z| \cdot \frac{|z|+2|c|}{1+2|c| \cdot|z|}\right]}
\end{gathered}
$$

where $c=\frac{\gamma_{1} \cdot a_{2}^{1}+\ldots \gamma_{n} a_{2}^{n}}{\left|\gamma_{1} \cdot \gamma_{2} \cdot \ldots \gamma_{n}\right|}$

$=\frac{-1}{3\left|\gamma_{1} \cdot \gamma_{2} \cdot \ldots \gamma_{n}\right|}\left[\frac{\gamma_{1}}{2 v_{1}+3}+\frac{\gamma_{2}}{2 v_{2}+3}+\ldots+\frac{\gamma_{n}}{2 v_{n}+3}\right]$

then for every $\beta \in \mathbb{C}, \operatorname{Re} \beta \geq b$ the function

$$
H(z)=\left\{\beta \int_{0}^{z} t^{\beta-1} \cdot\left[u_{v_{1}}^{\prime}(t)\right]^{\gamma_{1}} \cdot \ldots\left[u_{v_{n}}^{\prime}(t)\right]^{\gamma_{n}}\right\}^{\frac{1}{\beta}} d t
$$

is univalent

Proof:

Let the function $h_{1}$ defined by

$$
h_{1}(z)=\int_{0}^{z}\left[u_{v_{1}}^{\prime}(t)\right]^{\gamma_{1}} \cdot\left[u_{v_{2}}^{\prime}(t)\right]^{\gamma_{2}} \cdot \ldots \cdot\left[u_{v_{n}}^{\prime}(t)\right]^{\gamma_{n}} d t
$$

and the function $p$ defined by

$$
p(z)=\frac{1}{\left|\gamma_{1} \cdot \gamma_{2} \cdot \ldots \cdot \gamma_{n}\right|} \cdot \frac{h_{1}^{\prime \prime}(z)}{h_{1}^{\prime}(z)}
$$

After some calculus we have that:

$$
p(z)=\frac{\gamma_{1}}{\left|\gamma_{1} \cdot \gamma_{2} \cdot \ldots \gamma_{n}\right|} \cdot \frac{u_{v_{1}}^{\prime \prime}(z)}{u_{v_{1}}^{\prime}(z)}+\ldots+\frac{\gamma_{1}}{\left|\gamma_{1} \cdot \gamma_{2} \cdot \ldots \gamma_{n}\right|} \cdot \frac{u_{v_{n}}^{\prime \prime}(z)}{u_{v_{n}}^{\prime}(z)}
$$


D. Breaz, C.-I. Tănase - Applications of Bessel and Struve functions ...

Using relation (15) and (16) we obtain that

$$
|p(z)|<1,(\forall) z \in U
$$

and

$$
p(0)=\frac{\gamma_{1} \cdot a_{2}^{1}+\ldots \gamma_{n} \cdot a_{2}^{n}}{\left|\gamma_{1} \cdot \ldots \cdot \gamma_{n}\right|}=c
$$

where

$$
a_{2}^{i}=\frac{-1}{3\left(2 v_{i}+3\right)}
$$

for all $i \in\{1,2, \ldots, n\}$.

Applying Remark 1.1 for the function $p$ we obtain

$$
|p(z)| \leq \frac{|z|+2|c|}{1+2|c| \cdot|z|},(\forall) z \in U .
$$

Then

$$
\begin{gathered}
\frac{1}{\left|\gamma_{1} \cdot \gamma_{2} \cdot \ldots \cdot \gamma_{n}\right|} \cdot \frac{h_{1}^{\prime \prime}(z)}{h_{1}^{\prime}(z)} \leq \frac{|z|+2|c|}{1+2|c| \cdot|z|}, \quad(\forall) z \in U \\
\Rightarrow \frac{1}{\left|\gamma_{1} \cdot \gamma_{2} \cdot \ldots \cdot \gamma_{n}\right|} \cdot \frac{1-|z|^{2 b}}{b} \cdot\left|\frac{z h_{1}^{\prime \prime}(z)}{h_{1}^{\prime}(z)}\right| \leq\left[\frac{1-|z|^{2 b}}{b} \cdot|z| \cdot \frac{|z|+2|c|}{1+2|c| \cdot|z|}\right] \quad(\forall) z \in U . \\
\Rightarrow \frac{1-|z|^{2 b}}{b} \cdot\left|\frac{z h_{1}^{\prime \prime}(z)}{h_{1}^{\prime}(z)}\right| \leq\left|\gamma_{1} \cdot \gamma_{2} \cdot \ldots \cdot \gamma_{n}\right| \cdot \max _{|z| \leq 1}\left[\frac{1-|z|^{2 b}}{b} \cdot|z| \cdot \frac{|z|+2|c|}{1+2|c| \cdot|z|}\right]
\end{gathered}
$$

Using relation (17) we obtain that

$$
\frac{1-|z|^{2 b}}{b} \cdot\left|\frac{z h_{1}^{\prime \prime}(z)}{h_{1}^{\prime}(z)}\right| \leq 1(\forall) z \in U
$$

From Theorem 1.1 results that $H$ is univalent.

Corollary 2.1. Let $\alpha, \gamma \in \mathbb{C}, \operatorname{Re} \alpha=b>0, u_{v}$ function.

If

$$
\left|\sum_{n=0}^{\infty} \frac{n(n+1) \cdot z^{n-1}}{4^{n} \Gamma\left(n+\frac{3}{2}\right) \cdot \Gamma\left(v+n+\frac{3}{2}\right)}\right| \leq\left|\sum_{n=0}^{\infty} \frac{(n+1) \cdot z^{n}}{4^{n} \Gamma\left(n+\frac{3}{2}\right) \cdot \Gamma\left(v+n+\frac{3}{2}\right)}\right|,(\forall) z \in U .
$$


D. Breaz, C.-I. Tănase - Applications of Bessel and Struve functions ...

and

$$
|\gamma| \leq \frac{1}{\max _{|z| \leq 1}\left[\frac{1-|z|^{2 b}}{b} \cdot|z| \cdot \frac{|z|+2|c|}{1+2|c| \cdot|z|}\right]}
$$

where $c=\frac{-\gamma_{1}}{\left|\gamma_{1}\right| \cdot 3 \cdot(2 v+3)}$

then for every $\beta \in \mathbb{C}, \operatorname{Re} \geq b$ the function

$$
H(z)=\left\{\beta \int_{0}^{z} t^{\beta-1} \cdot\left[f_{v}^{\prime}(t)\right]^{\gamma}\right\}^{\frac{1}{\beta}} d t
$$

is univalent.

For $v=0$ the Corollary 2.1 will become:

Let $\alpha, \gamma \in \mathbb{C}, \operatorname{Re} \alpha=b>0, u_{0}$ Struve function.

If

$$
\left|\sum_{n=0}^{\infty} \frac{(n !)^{2} \cdot n \cdot(n+1) \cdot 2^{2 n+2} \cdot z^{n-1}}{((2 n+1) !)^{2}}\right| \leq\left|\sum_{n=0}^{\infty} \frac{(n !)^{2} \cdot(n+1) \cdot 2^{2 n+2} \cdot z^{n}}{((2 n+1) !)^{2}}\right|,(\forall) z \in U .
$$

and

$$
|\gamma| \leq \frac{1}{\max _{|z| \leq 1}\left[\frac{1-|z|^{2 b}}{b} \cdot|z| \cdot \frac{9 \cdot|z|+2}{9+2 \cdot|z|}\right]}
$$

then for every $\beta \in \mathbb{C}, \operatorname{Re} \geq b$ the function

$$
H(z)=\left\{\beta \int_{0}^{z} t^{\beta-1} \cdot\left[u_{0}^{\prime}(t)\right]^{\gamma}\right\}^{\frac{1}{\beta}} d t
$$

is univalent.

Theorem 2.2. Let $\alpha, \gamma_{i} \in \mathbb{C}, \operatorname{Re} \alpha=b>0, h_{v_{i}}$ functions $h_{v_{i}}(z)=z+b_{2}^{1} z^{2}+\ldots n \in$ $\mathbb{N}^{*}, i \in\{1,2, \ldots, n\}$. If

$$
\left|\frac{h_{v_{i}}^{\prime \prime}(z)}{h_{v_{i}}^{\prime}(z)}\right|<1,(\forall) z \in U,(\forall) i \in\{1,2, \ldots\}
$$

and

$$
\frac{\left|\gamma_{1}\right|+\left|\gamma_{2}\right|+\ldots+\left|\gamma_{n}\right|}{\left|\gamma_{1} \cdot \gamma_{2} \cdot \ldots \cdot \gamma_{n}\right|} \leq 1
$$




$$
\left|\gamma_{1} \cdot \gamma_{2} \cdot \ldots \cdot \gamma_{n}\right| \leq \frac{1}{\max _{|z| \leq 1}\left[\frac{1-|z|^{2 b}}{b} \cdot|z| \cdot \frac{|z|+2|c|}{1+2|c| \cdot|z|}\right]}
$$

where $c=\frac{\gamma_{1} \cdot b_{2}^{1}+\ldots \gamma_{n} b_{2}^{n}}{\left|\gamma_{1} \cdot \gamma_{2} \cdot \ldots \gamma_{n}\right|}$

$=\frac{-1}{4\left|\gamma_{1} \cdot \gamma_{2} \cdot \ldots \gamma_{n}\right|}\left[\frac{\gamma_{1}}{1+v_{1}}+\frac{\gamma_{2}}{1+v_{2}}+\ldots+\frac{\gamma_{n}}{1+v_{n}}\right]$

then for every $\beta \in \mathbb{C}, \operatorname{Re} \beta \geq b$ the function

$$
T(z)=\left\{\beta \int_{0}^{z} t^{\beta-1} \cdot\left[h_{v_{1}}^{\prime}(t)\right]^{\gamma_{1}} \cdot \ldots \cdot\left[h_{v_{n}}^{\prime}(t)\right]^{\gamma_{n}}\right\}^{\frac{1}{\beta}} d t
$$

is univalent.

Proof:

Let the function $t$ defined by

$$
t(z)=\int_{0}^{z}\left[h_{v_{1}}^{\prime}(t)\right]^{\gamma_{1}} \cdot\left[h_{v_{2}}^{\prime}(t)\right]^{\gamma_{2}} \cdot \ldots \cdot\left[h_{v_{n}}^{\prime}(t)\right]^{\gamma_{n}} d t
$$

and the function $p$ defined by

$$
p(z)=\frac{1}{\left|\gamma_{1} \cdot \gamma_{2} \cdot \ldots \cdot \gamma_{n}\right|} \cdot \frac{t^{\prime \prime}(z)}{t^{\prime}(z)}
$$

After some calculus we have that:

$$
p(z)=\frac{\gamma_{1}}{\left|\gamma_{1} \cdot \gamma_{2} \cdot \ldots \cdot \gamma_{n}\right|} \cdot \frac{h_{v_{1}}^{\prime \prime}(z)}{h_{v_{1}}^{\prime}(z)}+\ldots+\frac{\gamma_{1}}{\left|\gamma_{1} \cdot \gamma_{2} \cdot \ldots \cdot \gamma_{n}\right|} \cdot \frac{h_{v_{n}}^{\prime \prime}(z)}{h_{v_{n}}^{\prime}(z)}
$$

Using relation (22) and (23) we obtain that

$$
|p(z)|<1,(\forall) z \in U
$$

and

$$
p(0)=\frac{\gamma_{1} \cdot b_{2}^{1}+\ldots+\gamma_{n} \cdot b_{2}^{n}}{\left|\gamma_{1} \cdot \ldots \cdot \gamma_{n}\right|}=c
$$

where

$$
b_{2}^{i}=\frac{-1}{4\left(1+v_{i}\right)}
$$


D. Breaz, C.-I. Tănase - Applications of Bessel and Struve functions ...

for all $i \in\{1,2, \ldots, n\}$

Applying Remark 1.1 for the function $p$ we obtain

$$
|p(z)| \leq \frac{|z|+2|c|}{1+2|c| \cdot|z|},(\forall) z \in U
$$

Then

$$
\begin{gathered}
\frac{1}{\left|\gamma_{1} \cdot \gamma_{2} \cdot \ldots \cdot \gamma_{n}\right|} \cdot \frac{t^{\prime \prime}(z)}{t^{\prime}(z)} \leq \frac{|z|+2|c|}{1+2|c| \cdot|z|}, \quad(\forall) z \in U \\
\Rightarrow \frac{1}{\left|\gamma_{1} \cdot \gamma_{2} \cdot \ldots \cdot \gamma_{n}\right|} \cdot \frac{1-|z|^{2 b}}{b} \cdot\left|\frac{z t^{\prime \prime}(z)}{t^{\prime}(z)}\right| \leq\left[\frac{1-|z|^{2 b}}{b} \cdot|z| \cdot \frac{|z|+2|c|}{1+2|c| \cdot|z|}\right] \quad(\forall) z \in U . \\
\Rightarrow \frac{1-|z|^{2 b}}{b} \cdot\left|\frac{z t^{\prime \prime}(z)}{t^{\prime}(z)}\right| \leq\left|\gamma_{1} \cdot \gamma_{2} \cdot \ldots \cdot \gamma_{n}\right| \cdot \max _{|z| \leq 1}\left[\frac{1-|z|^{2 b}}{b} \cdot|z| \cdot \frac{|z|+2|c|}{1+2|c| \cdot|z|}\right]
\end{gathered}
$$

Using relation (24) we obtain that

$$
\frac{1-|z|^{2 b}}{b} \cdot\left|\frac{z t^{\prime \prime}(z)}{t^{\prime}(z)}\right| \leq 1(\forall) z \in U
$$

From Theorem 1.1 results that $T$ is univalent.

Corollary 2.2. Let $\alpha, \gamma_{1} \in \mathbb{C}, \operatorname{Re} \alpha=b>0, h_{v}$ function. If

$$
\left|\sum_{n=0}^{\infty} \frac{n(n+1) \cdot z^{n-1}}{n ! \cdot 4^{n} \cdot \Gamma(n+v+1)}\right| \leq\left|\sum_{n=0}^{\infty} \frac{(n+1) \cdot z^{n}}{n ! \cdot 4^{n} \cdot \Gamma(n+v+1)}\right|,(\forall) z \in U .
$$

and

$$
\left|\gamma_{1}\right| \leq \frac{1}{\max _{|z| \leq 1}\left[\frac{1-|z|^{2 b}}{b} \cdot|z| \cdot \frac{|z|+2|c|}{1+2|c| \cdot|z|}\right]}
$$

where $c=\frac{-\gamma_{1}}{4\left|\gamma_{1}\right|(1+v)}$ then for every $\beta \in \mathbb{C}, \operatorname{Re} \beta \geq b$ the function

$$
T(z)=\left\{\beta \int_{0}^{z} t^{\beta-1} \cdot\left[h_{v}^{\prime}(t)\right]^{\gamma_{1}}\right\}^{\frac{1}{\beta}} d t
$$

is univalent 
D. Breaz, C.-I. Tănase - Applications of Bessel and Struve functions ...

For $v=0$ the Corollary 2.2 will become:

Let $\alpha, \gamma_{1} \in \mathbb{C}, \operatorname{Re} \alpha=b>0, h_{0}$ function. If

$$
\left|\sum_{n=0}^{\infty} \frac{n(n+1) \cdot z^{n-1}}{(n !)^{2} \cdot 4^{n}}\right| \leq\left|\sum_{n=0}^{\infty} \frac{(n+1) \cdot z^{n}}{(n !)^{2} \cdot 4^{n}}\right|,(\forall) z \in U .
$$

and

$$
\left|\gamma_{1}\right| \leq \frac{1}{\max _{|z| \leq 1}\left[\frac{1-|z|^{2 b}}{b} \cdot|z| \cdot \frac{2|z|+1}{|z|+2}\right]}
$$

then for every $\beta \in \mathbb{C}, \operatorname{Re} \beta \geq b$ the function

$$
T(z)=\left\{\beta \int_{0}^{z} t^{\beta-1} \cdot\left[h_{0}^{\prime}(t)\right]^{\gamma_{1}}\right\}^{\frac{1}{\beta}} d t
$$

is univalent.

For $v=1$ the Corollary 2.2 will become:

Let $\alpha, \gamma_{1} \in \mathbb{C}, \operatorname{Re} \alpha=b>0, h_{1}$ function. If

$$
\left|\sum_{n=0}^{\infty} \frac{n(n+1) \cdot z^{n-1}}{n ! \cdot(n+1) ! \cdot 4^{n}}\right| \leq\left|\sum_{n=0}^{\infty} \frac{(n+1) \cdot z^{n}}{n ! \cdot(n+1) ! \cdot 4^{n}}\right|,(\forall) z \in U .
$$

and

$$
\left|\gamma_{1}\right| \leq \frac{1}{\max _{|z| \leq 1}\left[\frac{1-|z|^{2 b}}{b} \cdot|z| \cdot \frac{4|z|+1}{|z|+4}\right]}
$$

then for every $\beta \in \mathbb{C}, \operatorname{Re} \beta \geq b$ the function

$$
T(z)=\left\{\beta \int_{0}^{z} t^{\beta-1} \cdot\left[h_{1}^{\prime}(t)\right]^{\gamma_{1}}\right\}^{\frac{1}{\beta}} d t
$$

is univalent.

\section{REFERENCES}

[1] Becker J., Lőwnersche, Differentialgleichnung und quasikonform fortsetzbare schichte Functionen, J. Reine Angew. Math. 255, (1972), 23-43.

[2] Goluzin G.M., Geometriceskaia teoria funktii kompleksnogo peremenogo, Moscova, (1966). 
D. Breaz, C.-I. Tănase - Applications of Bessel and Struve functions ...

[3] Nehari Z., Conformal mapping, McGraw-Hill Book Comp., New York, (1952), (Dover. Publ.Inc., 1975).

[4] Orhan H.,Yagmur N., Geometric properties of generalized Struve functions, The Journal of "Alexandru Ioan Cuza" University from Iasi, (2014).

[5] Pascu N.N., An improvement of Becker's univalence criterion, Proceedins of the Commerative Session Simion Stoilow, Braşov, (1987), 43-48.

[6] Szász R., Kupán P.A., About the univalence of the Bessel functions, Studia.Univ."Babeş-Bolyai", Mathematica, Volume LIV, Number 1, March 2009

[7] Zhang, S.; Jin,J., Computation of Special Functions, A Wiley-Interscience Publication, John Wiley and Sons, Inc., New York,(1996).

Daniel Breaz,

"1 Decembrie 1918" University of Alba Iulia, N.Iorga Str., No. 11-13,

510009 Alba Iulia, Alba, Romania email: dbrea@uab.ro

Carmen-Ioana Tănase,

University of Piteşti,

Târgul din Vale Str., No. 1,

110040 Piteşti, Argeş, Romania 